\title{
MedienPädagogik
}

Zeitschrift für Theorie und Praxis der Medienbildung

www.medienpaed.com

ISSN 1424-3636

Jahrbuch Medienpädagogik 15:

Erziehungswissenschaftliche und medienpädagogische Online-Forschung:

Herausforderungen und Perspektiven

Herausgegeben von Johannes Fromme, Stefan Iske, Therese Leik, Steffi

Rehfeld, Jasmin Bastian, Manuela Pietraß und Klaus Rummler

\section{"There is much about Wraith that you do not know»}

\section{Medienbildungspotenziale eines literarischen Rollen- spiels zur Science-Fiction-Serie Stargate Atlantis}

Wolfgang Reißmann

\section{Zusammenfassung}

Aus kommunikationssoziologischer Sicht ist die in der Alltagspraxis von Individuen und Gruppen situierte Perspektivierungsarbeit eine Voraussetzung für Medienbildungsprozesse. Fan Fiction und transformatives Werkschaffen begünstigen solche Arbeiten, insofern ihre Praxis ein beständiges Oszillieren zwischen Vorlage und Neuschöpfung, verschiedenen medialen Formen, Vergangenheit und Gegenwart sowie diversen Beteiligungsrollen nahelegt. Im Mittelpunkt des Beitrags steht vor diesem Hintergrund ein literarisches Rollenspiel, das eine Gruppe von Fans auf Basis der Science-Fiction-Serie Stargate Atlantis initiiert hat und das im Rahmen einer Studie zu kooperativ verfassten transformativen Fanwerken medienethnografisch begleitet wurde. Exemplarisch werden Medienbildungspotenziale des Rollenspiels auf den Ebenen des Grenz-, Handlungs-, Wissens- und Biografiebezugs herausgearbeitet. Aus der Warte der Spezies der Wraith verfasst, nehmen die Spielerinnen und Spieler eine fundamentale Perspektivumkehrung vor und manövrieren spielend durch Herrschafts- und Machtverhältnisse. 


\title{
"There is much about Wraith that you do not know». Potentials for Media Education in a literary Role-playing Game for the Science Fiction Series Stargate Atlantis
}

\begin{abstract}
From the point of view of communication sociology, the perspectivation work situated in the everyday practice of individuals and groups is a prerequisite for media education processes. Fanfiction and transformative working favour perspectivation work, insofar as they suggest a constant oscillation between existing material and new creation, between various media forms, between past and present, as well as between various participation roles. Against this background, the contribution focuses on a literary role play initiated by a group of fans on the basis of the science fiction series Stargate Atlantis. This role play was accompanied by media ethnography as part of a study on cooperative transformative fan works. The role plays's potentials for media education are worked out exemplarily on the levels of "boundary reference», "action reference», "knowledge reference» and «biographical reference». Written from the perspective of the Wraith species, the players make a fundamental reversal of perspective and playfully navigate through relations of domination and power.
\end{abstract}

\section{Alltägliche Perspektivierungsarbeit als Basis für Medienbildung}

Medienbildung ist ein Ansatz, der in der deutschsprachigen Erziehungswissenschaft über die Abgrenzung zu konventionellen Lerntheorien sowie die Reformulierung bildungsphilosophischer Traditionen unter den Vorzeichen postmoderner und mediatisierter Lebensverhältnisse eingeführt ist. Ausgangspunkt ist die Annahme, dass ein rezentes Bildungsverständnis nicht (mehr) über Inhalte oder kanonisches Wissen zu definieren sei, sondern struktural auf die Flexibilisierung, Dezentrierung und Transformation von Selbstund Weltverhältnissen zu zielen habe. Insofern damit überhaupt 
Kompetenzen ${ }^{1}$ adressiert sind, dann vor allem die Kompetenz des Subjekts, mit Kontingenz, Unbestimmtheit, Pluralität und Heterogenität umzugehen. Das impliziert keine relativistische Position der Nichtfestlegung/-bewertung oder postmoderner Beliebigkeit, aber: «Unbestimmtheiten müssen einen Ort, besser mehrere Orte in unserem Denken erhalten (...)» (Jörissen und Marotzki 2009, 21).

Wenn Flexibilisierung, Dezentrierung und Transformation von Selbst- und Weltverhältnissen den bildungstheoretischen Zielhorizont spannen, dann lässt sich darauf aufbauend als Voraussetzung für Medienbildung eine in der Alltagspraxis der Individuen und Kollektive situierte Perspektivierungsarbeit ableiten. Bildung wird möglich, wenn Subjekte und Gruppen andere, ihnen bislang fremde Perspektiven und Weltsichten kennenlernen, sie sich in andere kulturelle Verortungen, Sinnhorizonte, Handlungs- und Verhaltensmuster einfühlen und selbst-reflexiv Stellung zu den so gemachten Erfahrungen beziehen.

Kommunikationssoziologisch sind Perspektivübernahme und -koordination anthropologische Techniken, ohne die Kommunikation zwischen sowie innerhalb von Menschen und von Menschen mit und über Medien nicht denkbar ist. Mindestens darf das für die Kommunikationszugänge angenommen werden, die sich dem Symbolischen Interaktionismus verpflichtet fühlen. Selbstverständlich aber ist nicht jede in kommunikativer Interaktion getätigte Perspektivübernahme schon bildungsrelevant. Bildungsrelevant wird

1 In der fachlichen Kontroverse haben Medienbildung und Medienkompetenz bekanntlich symptomatischen Charakter. Als eigenständige Strömung wurde Medienbildung recht scharf vom Begriff der Medienkompetenz abgegrenzt. Die Reduktion des Begriffs der Medienkompetenz auf lerntheoretische und entwicklungspsychologische Verkürzungen wird m.E. jedoch weder dessen kommunikations- und handlungstheoretischen Wurzeln gerecht noch der mit ihm seit den 1970er Jahren verbundenen handlungsorientierten Medienarbeit, deren Prinzipien nicht weniger auf das Ermöglichen von Unbestimmtheit, das Infragestellen des Bestehenden und die Anregung zur Gestaltung von Selbst- und Weltverhältnissen zielen. 
die (kommunikative) Konfrontation mit der sozialen und materiellen (Um-)Welt dann, wenn sie nicht auf routineartige Praxis und Vollzug beschränkt bleibt, sondern die geleistete Perspektivierungsarbeit den Subjekten als solche verfügbar wird, sie sie sich gewissermassen als Erfahrung kognitiv und/oder ästhetisch zum Gegenstand machen.

Beide Prozesse, Kommunikations- wie Bildungsprozesse, vollziehen sich unter den Bedingungen von Medialität. Das gilt für die Gegenwart wie für die Vergangenheit. In Prozessen der Medienbildung kann, muss es aber nicht um die Medien selbst gehen. Anregungsund Orientierungspotenziale speisen sich aus dem, was medial artikuliert ist, und was man mit und in Medien erleben kann (wenn man beispielsweise fernsieht, mit anderen spricht, spielt oder medienvermittelt zusammenarbeitet). Und sie speisen sich aus dem, wie Medien artikuliert sind und wie sie ihre eigene Geworden- und Gemachtheit als Medien erfahrbar machen (von Repräsentations- und Darstellungsmustern, über Kanalspezifika zu Game Play, InterfaceDesign oder Datenästhetik). Gleichsam zwischen dem (sich selbst bildenden) Subjekt und dem medialen Objekt als Gegenüber und Vermittler, sind Anregungs- und Orientierungspotenziale zudem in den Praktiken des Medienumgangs selbst eingelagert. Diese Praktiken werden in spezifischen Kulturen, Praxisgemeinschaften und sozialen Welten ausgebildet.

\section{Fan Fiction als spezifische Medienkultur}

Im Mittelpunkt dieses Beitrags stehen literarische Werke von Fans, die auf bereits bestehenden medialen Werken aufbauen. Diese modifizierten, erweiterten oder neuen Erzählungen werden Fan Fiction genannt. In ihren Schriften bedienen sich die Autorinnen und Autoren der Figuren, Narrationen und/oder Welten bekannter Werke. In «pairings» bringen sie beispielsweise bestehende oder selbst erfundene Figuren in romantischen und sexuellen Beziehungen zusammen. Im Subgenre der «Slash»-Geschichten sind gleichgeschlechtliche, häufig homoerotische Beziehungen zentraler Inhalt. 
Ein pejoratives Vorurteil reduziert Fan Fiction auf Sex-Geschichten. Das wird ihrer Vielfalt nicht gerecht. Ein euphemistisches Vorurteil rückt Fan Fiction per se in die Nähe von Gesellschafts-, Medienkritik und Identitätspolitik (ethnische Herkunft, Milieu, LGBQTI). Auch das lässt sich nicht verallgemeinern. Fan Fiction ist weder per se tiefschürfender oder oberflächlicher als die Vorlagen, auf die sie sich bezieht (zumal «Qualität» normativ erst einmal definiert werden müsste). Tatsache ist allerdings, dass Fan Fiction-Autorinnen und -Autoren bestehende Werke gern gegen den Strich rezipieren bzw. verfassen und sie diese anders machen.

Wenngleich heute mit digitalen Medien assoziiert und realisiert, ist Fan Fiction kein Produkt der Digitalisierung oder aktueller Mediatisierungsschübe. Sie durchzieht mindestens die Mediengeschichte der vergangenen 130 Jahre (Cuntz-Leng und Meintzinger 2015). Unter analogen Bedingungen waren Fanzines, postalische Netzwerke und Conventions die zentralen Verbreitungsmedien. Mit Internet und WWW sind Mailinglisten und Foren rasch wichtig geworden. Seit der Jahrtausendwende prägen neben unzähligen Tumblr-Blogs und privaten Seiten grosse Archivplattformen wie Archive of Our Own (Ao3), Fanfiction.net, FanFiktion.de oder Animexx.de die Fan Fiction-Landschaft.

Als eigenständige Medienkultur ist Fan Fiction am Kreuzungspunkt von literarischer Textproduktion und den diversen Fandoms zu lokalisieren, auf die sich diese Produktion bezieht. Soll heissen: Zum einen sind das Interesse am Schreiben und das transformative Werkschaffen Ausgangspunkt für Vergemeinschaftung. Zum anderen sind die Autorinnen und Autoren selbst Fans bestimmter medialer Produkte und Franchises. Entsprechend identifizieren sie sich mit der (über Fan Fiction hinausgehenden) Fankultur um Harry Potter, Star Trek, Yu-Gi-Oh etc.

Fan Fiction reiht sich in die Liste der Phänomene ein, die in die «remix culture» und die "participatory culture» subsumiert werden. Folgt man den «new literacy studies» (Knobel und Lankshear 
2008) und den akademischen Vorreitern der Partizipationskultur, sind Spielen, Simulieren, Aneignen, Multitasking, verteilte Kognition, kollektive Intelligenz, Beurteilen, transmediales Navigieren, Networking und Verhandeln (z. B. Jenkins et al. 2006, 4) Basisfähigkeiten, um in digitalen Medienkulturen zu bestehen. In diesem Sinn ist Fan Fiction ein Lern- und Trainingsfeld unterschiedlichster Fähigkeiten. Hervorgehoben werden in der bestehenden Forschung die Potenziale für die Ausdifferenzierung von (schrift-/fremd-)sprachlichen und narrativen Fähigkeiten sowie die für Fan Fiction charakteristische Einbindung in ein Peer-to-Peer-Mentoring, etwa über die verbreitete Zusammenarbeit mit so genannten «beta reader(s)» (zumeist befreundete Autorinnen und Autoren), die als Lektoren und Editoren am Produkt mitwirken (Black 2008).

In einer bildungsbezogenen Sicht sind vor allem jedoch die Potenziale von Fan Fiction in den Mittelpunkt zu rücken, die Perspektivierungsarbeiten und dabei mögliche selbst-reflexive Erfahrungen begünstigen. Aus einer laufenden Studie, deren Ergebnisse sich für eine bildungsbezogene Relektüre anbieten ${ }^{2}$, lassen sich mindestens vier Relationen ableiten, die Perspektivierungsarbeiten nahelegen:

- Vorlage/Neuschöpfung: Fan Fiction-Autorinnen und -Autoren wechseln permanent zwischen Vorlage («Canon») und darauf aufbauender Fantasie («Fanon»). Sie eignen extensiv und intensiv Wissensordnungen zum jeweiligen Fandom an, die in der eigentätigen Konstruktion und Produktion von Figuren, Beziehungen, Handlungssträngen und Welten zugleich überschritten und überschrieben werden.

- Form/Form: Fan Fiction-Autorinnen und -Autoren sind in ihrem Alltag mit Medienwechseln unterschiedlichster Art konfrontiert:

2 Die Studie ist nicht in bildungsbezogener Absicht initiiert, sondern Bestandteil des interdisziplinären Teilprojekts Bo7 «Medienpraktiken und Urheberrecht» des Sonderforschungsbereichs «Medien der Kooperation» an der Universität Siegen. Im Mittelpunkt stehen transformative und kooperative Praktiken von Fans, deren rechtliche Bewertung sowie rechtspolitische Überlegungen zur Ausbalancierung urheberrechtlicher Interessen verschiedener Akteursgruppen. 
z.B. in der Überführung oft dominant audiovisuell geprägter Vorlagen in die Form der Literatur; in Übersetzungsarbeiten, weil in englischer Sprache rezipiert und/oder geschrieben wird; in der mehrgleisigen Publikation ihrer Geschichten in verschiedenen Plattformen mit verschiedenen Klassifikationssystemen, Interfaces/Layouts und Plattformpolitiken; als Leserinnen, Leser, Autorinnen und Autoren im Wechsel zwischen spezifischen Schreibgenres.

- Früher/Heute: Fan Fiction-Autorinnen und -Autoren begegnen in diachroner Hinsicht ihren bereits verfassten und publizierten Geschichten und damit ihren vergangenen Autor-Selbsten und Fantasien.

- Rolle/Rolle: Fan Fiction-Autorinnen und -Autoren wechseln zwischen verschiedenen Beteiligungsrollen: Sie sind «konsumierende) und produzierende Fans. Sie sind Leserinnen, Leser, Autorinnen, Autoren, Lektorinnen, Lektoren sowie Kommentatorinnen und Kommentatoren. Institutionalisierte bzw. verfestigte Bearbeitungs- und Interaktionsschlaufen produzieren Feedback.

Diese vier in der Alltagspraxis und den Umgangspraktiken angelegten Relationen des «Doing» Fan Fiction erzwingen weder umfassende Transformationen im Selbst- und Weltverhältnis, noch, als Vorstufe und Bedingung, bleibende und bewegende Erfahrungen, die aus der alltäglichen Perspektivierungsarbeit als Fan und AutorIn resultieren. Die praktische Grundstruktur von Fan Fiction macht solche Prozesse, so die These, aber wahrscheinlich. Am Beispiel eines literarischen Rollenspiels und der dabei produzierten Texte soll das im Folgenden illustriert werden. 


\section{Mit den Augen der Wraith sehen: Ein Rollenspiel zu Stargate Atlantis}

Eine der Teilstudien des genannten Projekts fokussiert eine Roleplay/RP-Gruppe, die von Februar bis August 2017 medienethnografisch begleitet wurde.

In ihrer literarischen Gestalt ist RP eine Form des kollektiven Schreibens, in der - je nach Blickwinkel - schreibende Spielerinnen und Spieler oder spielende Autorinnen und Autoren gemeinsam Geschichten kreieren. Im Projekt betrachten wir literarische RPs als eine (per se) kollektive Form bzw. Verwandte von Fan Fiction; neben Partner- und Mitmachgeschichten; neben Schreibformen, die dem traditionalen Konzept des Individualautors verbunden bleiben; neben spezifischen Rahmungen wie Schreib-Wettbewerben (die sich wiederum auf die diversen Schreibformen beziehen). ${ }^{3}$ RPs werden vornehmlich in chatförmigen Medienumgebungen gespielt, aber auch per Forum oder Messenger. Die Spielenden sind für einen oder mehrere Charaktere verantwortlich, aus deren Perspektive Erzähltext und Figurenrede verfasst werden. Im Dialog der Figuren entsteht aufeinander aufbauend so der gemeinsame Text.

Die Grenzen zwischen eher literarischen RPs und textbasierten MUDs (Multi User Dungeons) und Pen \& Paper-Rollenspielen verlaufen fliessend. Beim literarischen RP stehen eher die Story- und Charakterentwicklung im Mittelpunkt. Spezifische Canon- und Fanon-Vorgaben begrenzen dabei das Geschehen. MUDs und Pen \& Paper-Rollenspiele bestimmen sich stärker über spezifische Spielregeln (z.B. in der Interaktion der Charaktere oder Aktions- und Angriffsvarianten).

3 Diese deduktive Subsumtion läuft den Alltagsheuristiken der Fans gelegentlich zuwider. Oft werden RP (kollektiv, Spielen als zentraler Referenzpunkt) und Fan Fiction (eher individual, Schreiben als zentraler Referenzpunkt) als abgegrenzte Tätigkeitsfelder bestimmt. 


\subsection{Stargate Atlantis, die Wraith und die Gruppe}

Das begleitete RP ist im Stargate Fandom angesiedelt und handelt von der Spezies der Wraith ${ }^{4}$, die in der militärischen Science-FictionSerie Stargate Atlantis (5 Staffeln; 2004-2008) bedeutsam ist. Stargate Atlantis ist ein Ableger der Serie Stargate - Kommando SG 1 (10 Staffeln; 1997-2007), die selbst auf den 1994 veröffentlichten Kinofilm Stargate (Regie: Roland Emmerich) zurückgeht. Stargate Atlantis baut auf der Entdeckung der verloren geglaubten Stadt der Antiker in der 7. Staffel von Stargate SG 1 auf. Sie beschreibt die daraufhin ins Leben gerufene Expedition in die Pegasus-Galaxie, die seit einem Krieg zwischen Antiker und Wraith vor über 10.000 Jahren von den Wraith dominiert wird. Letztere sind antagonistisch als permanente Bedrohung der Atlantis Expedition eingeführt.

Zum Basis-Canon-Wissen gehört, dass die insektenartige Spezies in Hives organisiert ist, die von Königinnen bzw. Queens hierarchisch regiert werden. Während die Königinnen weiblich sind (nur in Ausnahmefällen gibt es männliche Hivemaster), sind alle anderen Wraith männlich. Sie differenzieren sich in Blades als kämpfende Akteure sowie Clevermen als technisch-wissenschaftliches Personal, das für die Raumschiffe und die technische Infrastruktur verantwortlich ist.

Die Serie Stargate Atlantis ist aus der Sicht der handelnden Menschen erzählt, deren Wissen zu den Wraith beschränkt ist. Wraith werden, z.B. in Angriffs- und Kampfszenen, meist stereotyp gezeichnet. Gleichzeitig sind in der Vorlage Momente angelegt, die die Fan Community beschäftigen und neuralgisch für Fan-Extensionen zu den Wraith sind. So etwa Episode 7 der 3. Staffel mit dem Titel «Ein ungewöhnlicher Verbündeter» (deutsch) bzw. «Common Ground» (englischer Originaltitel). Lieutenant Colonel John Sheppard wird hier gemeinsam mit einem Wraith, den er Todd nennt, von Acastus Kolya (vom Volk der Genii, in mehreren Folgen Sheppards

4 Da es sich um eine Spezies handelt, in der die Abhängigkeit vom Kollektiv zentral ist, steht das Wort «Wraith» gleichermassen für Singular und Plural. 
Gegenspieler) gefangen gehalten. Um sich zu befreien, verbünden sich Sheppard und Todd.

Wraith ernähren sich von Menschen. In einem Feeding genannten Prozess nehmen sie ihnen Lebenskraft. Todd stärkt sich an John Sheppard und kann so Kolyas Männer im Kampf bezwingen, die ihm dann wiederum als Energiequelle dienen. So ist er fähig, dem ohnehin und durch das Feeding weiter geschwächten John Sheppard das sogenannte Gift of Life zu spenden. Wraith verfügen über diese Fähigkeit, physische Wunden und Krankheiten heilen zu können. In den Genuss dieses Geschenks kommen aber nur «Brüder» und enge Verbündete. Es ist also ein aussergewöhnlicher Vorgang, dass ein Wraith einem Menschen Gift of Life gibt. Auch Sheppard hat nicht ernsthaft daran geglaubt, dass Todd Wort hält. Im Rahmen der Episode kommt es zu Annäherungen der beiden Charaktere. Sheppard ist sich sicher, dass ihn sein Team nicht im Stich lassen wird. Wraith sind ohne ihr Kollektiv, den Hive, nicht denkbar. Diese Parallele veranlasst Todd zu dem Kommentar, dass Sheppard einem Wraith ähnlicher sei als er denke, woraufhin Sheppard entgegnet, dass er nicht sicher sei, ob ihm das gefallen soll. Darauf Todd im englischen Original:

«There is much about Wraith that you do not know Sheppard».

Dieser Satz aus Episode 03.07 kann als Leitmotiv der RP-Gruppe betrachtet werden. Im Vergleich zur Vorlage des Serien-Canon nimmt die Gruppe eine fundamentale Perspektivumkehrung vor. In ihrem RP sind alle Hauptcharaktere Wraith-OCs. Das Kürzel OC steht für «original character», d.h. für selbst kreierte und gestaltete Figuren. Das RP führt die Wraith-Figuren aus der relativen Ges(ch)ichtslosigkeit des Canon heraus, indem es ihnen Individualität, komplexe Beziehungsstrukturen und narrative Tiefe verleiht.

Mitglieder der Autorinnen- und Autoren- bzw. Spielgruppe sind Natalie (29 Jahre zum Beginn der Beobachtung), Mario (31 Jahre), 
Nadja (24 Jahre) $)^{5}$ und Svenja (26 Jahre). Mario, Natalie und Svenja sind befreundet und wohnen in einer mittelgrossen deutschen Stadt. Nadja ist Kanadierin und lebt dort ebenfalls in einer mittelgrossen Stadt. Svenja ist zugleich studentische Mitarbeiterin im Projekt und seit vielen Jahren Fan Fiction-Autorin und Rollenspielerin. ${ }^{6}$ Die Ursprungsgruppe hat sich über ein RP im Star Trek Fandom kennengelernt. Nach einer anfänglichen Beobachtungs- und Einfindungsphase nimmt Svenja aktiv am Schreib- und Spielprozess der Gruppe teil. Das Fandom ist neu für sie.

Gespielt wird das RP translokal in "virtual rooms» der ChatPlattform Chatzy. Schreib- und Spielsprache ist Englisch. Im Nachgang archiviert Mario den jeweils entstandenen Textkorpus in bereinigter Form (u.a. löscht er die in Klammern ausgezeichnete Meta-Kommunikation während des Spielens) als RP Log im Stash, ein Zwischenspeicher der Plattform Deviant Art. Diese Logs sind nur per Link einsehbar. Im Beobachtungszeitraum entstehen ca. 200 RPs (folgend: Episoden genannt), die in wechselnder Besetzung gespielt werden. Die einzelnen Episoden entstehen meist innerhalb von fünf bis zehn Stunden. In seltenen Fällen wird unterbrochen und eine Episode am Folgetag zu Ende gebracht.

Methodisch folgt die Teilstudie den Prinzipien der digitalen Ethnografie (Hine 2015), mit einem autoethnografischen Fokus auf die während der aktiven Partizipation gemachten Erfahrungen. Zugleich besteht eine Besonderheit der Studie im beständigen Wechsel zwischen autoethnografischer Teilnahme und Analyse im Team.

5 Natalie, Mario und Nadja sind anonymisierte Namen. Svenja wird als studentische Mitarbeiterin des Projekts nicht anonymisiert.

6 Ohne Svenja Kaisers Engagement und Teilhabe wäre diese Studie nicht möglich gewesen. Ihr gebührt an dieser Stelle ein ebenso grosser Dank wie allen Gruppenmitgliedern, die uns Einblick in ihre Fantasiewelt und ihren Spielalltag gaben. Gleiches gilt für Lisa Theresa Schröter und Moritz Stock, die zusammen mit Svenja Kaiser und Wolfgang Reißmann den Kern der Auswertungs- und Interpretationsgruppe bilden. 
Anhand der Protokolle und der Screenshots zu den Spielsessions ,partizipiert' die Interpretationsgruppe in einem nachträglichen Akt gleichsam sekundär am Spielgeschehen. Dieses spezifische Arrangement hat sich als produktive Quelle wechselseitiger Befremdung erwiesen. Die Primärerfahrungen der Ethnografin bilden den Ausgangspunkt. Nicht weniger relevant sind jedoch die Reaktionen und Perspektiven der Mitglieder der Interpretationsgruppe, die /von aussen` an das Material herangetragen helfen, vermeintliche Selbstverständlichkeiten zu hinterfragen.

\section{2 «Welcome to Hive Fairweather»: Ausgewählte Medienbil- dungspotenziale des RP}

In ihrer Einführung in den Medienbildungsansatz explizieren Jörissen und Marotzki (2009, 31-37) vier Dimensionen einer strukturalen Medienanalyse. Die vier lebensweltlichen Orientierungen des Grenzbezugs (Reflexion auf Grenzen der Rationalität und elementarer Existenzbedingungen), des Handlungsbezugs (Reflexion auf ethische und moralische Grundsätze), des Wissensbezugs (Reflexion auf Bedingungen und Grenzen des Wissens) sowie des Biografiebezugs (Reflexion auf eigene Identität und ihrer biografischen Bedingungen) leiten die folgenden Ausführungen und bildungsbezogenen Relektüren der Daten interpretativ an.

Gegenstand der Analyse sind die Texte ausgewählter RP-Episoden, an denen exemplarisch Medienbildungspotenziale herausgearbeitet werden. Das Augenmerk liegt primär auf der Was-Perspektive, aus Gründen des Platzmangels nur sekundär auf der Wie-Perspektive (zur Unterscheidung siehe oben). Nicht minder relevante Aspekte der formal-dramaturgischen Gestaltung z.B. der Episoden«Opener», Episoden-Abschlüsse, Figurenwechsel, der Einführung von Charakteren, der Fokalisierung, sowie die detaillierte Rekonstruktion von Canon- und Fanon-Referenzen im Textkorpus oder die Differenz von Spieler- und Charakterwissen können nur am Rande behandelt werden. Tabelle 1 gibt einen Überblick zu den folgend thematisierten OCs. 


\begin{tabular}{|l|l|}
\hline Hives & Figuren \\
\hline $\begin{array}{l}\text { Fairweather = Hive, auf dem zumeist } \\
\text { gespielt wird. Hier haben Blades das } \\
\text { Sagen. Den thematisierten Episoden } \\
\text { vorausgegangen ist eine Rebellion der } \\
\text { unterdrückten Clevermen. }\end{array}$ & $\begin{array}{l}\text { Wallflower (gespielt von Natalie) = die } \\
\text { noch junge Königin des Hives Fairwea- } \\
\text { ther. } \\
\text { Cinder (gespielt von Mario) = Clever- } \\
\text { man, der sich mit Luckless angefreun- } \\
\text { det hat. } \\
\text { Luckless (gespielt von Svenja) = Clever- } \\
\text { man, dessen Hive vernichtet und der } \\
\text { neu in den Hive Fairweather aufgenom- } \\
\text { men wurde. }\end{array}$ \\
\hline $\begin{array}{l}\text { Cyclone = Hive, der während der Rebel- } \\
\text { lion eine vermittelnde Rolle hat. Sein } \\
\text { vorübergehender Hivemaster, Caprice, } \\
\text { nimmt die von Fairweather geflüchte- } \\
\text { ten Clevermen auf, unterstützt Wallflo- } \\
\begin{array}{l}\text { wer aber auch dabei, die angespannte } \\
\text { Situation zu klären und eine Rückkehr } \\
\text { der Clevermen zu ermöglichen. }\end{array}\end{array}$ & $\begin{array}{l}\text { Derman, der als Abgesandter des Hives } \\
\text { Cyclone mit Wallflower Kontakt auf- }\end{array}$ \\
\hline
\end{tabular}

Tab. 1.: Überblick zu den im Fliesstext genannten Hives und Figuren. Quelle: Eigene Darstellung.

\section{Grenzbezug: Was is(s)t der Wraith?}

Die Welt aus der Sicht der Wraith zu beschreiben, erfordert das Einfühlen in die Beschaffenheit und die Lebensbedingungen einer anderen Spezies. Ein Beispiel hierfür ist das Sujet des Feeding. Wie erwähnt ernähren sich Wraith von Menschen. Sie betrachten Menschenvölker als ihre Herden. Von Zeit zu Zeit schwärmen sie aus, um ihre Vorräte aufzufrischen (Culling). Teilweise leben Menschen aber auch an Bord der Raumschiffe. Diese Worshippers beten die Wraith an. Sie dienen ihnen und werden im Gegenzug am Leben gelassen. Mithin betrachten Wraith Worshippers als Kuscheltiere bzw. "pets». ${ }^{7}$ Nicht alle Hives akzeptieren sie auf ihren Schiffen, weil sie Schwierigkeiten haben mit der Ambivalenz, diese zugleich als Wesen mit Gefühlen und als Nahrung zu betrachten.

7 Siehe zu dieser Ambivalenz z.B. den Eintrag «wraith worshipper» des Stargate Wiki: http://stargate.wikia.com/wiki/Wraith_worshipper. 
Auch im RP sind Nahrung und Feeding immer wieder Thema, meist beiläufig, manchmal zentral gestellt. So in der Episode «Deepwater convinces Queen Wallflower to feed» vom 24.06.2017, die auf dem Hive Fairweather spielt. Vorausgegangen ist eine Rebellion der Clevermen gegen die Blades. Diese Rebellion ist im Beobachtungszeitraum ein plotbestimmendes Ereignis (mehr dazu im nächsten Abschnitt). Ein Grossteil der Clevermen hat das Schiff verlassen und die junge Königin Wallflower ${ }^{9}$ gerät in Folge fortwährender Attacken mit ihrem dezimierten Hive in Bedrängnis. Zusätzlich werden die Vorräte knapp und die Konkurrenz mit anderen Hives verstärkt den Druck. Wallflower (gespielt von Natalie), fühlt sich, so stellt sich im Laufe der Episode heraus, für die schlechte Lage ihres Hives verantwortlich und versagt sich angesichts der angespannten Lage zu essen: «Feeding herself while her men were hungry and restricted didn't seem right».

Deepwater (gespielt von Nadja) ist ein abgesandter Cleverman des verbündeten Hives Cyclone, auf dem Wallflowers Clevermen Zuflucht gefunden haben. Er soll vermitteln und die wenigen nach der Rebellion auf dem Schiff verbliebenen Clevermen anleiten. Ein Handlungsstrang der Episode besteht in der Entwicklung einer vertrauensvollen Beziehung der beiden mit offenem Ausgang. Die Rahmenhandlung ist, dass er sich um Gesundheit und Kraft der Königin sorgt. Deepwater möchte Wallflower dazu bewegen, sich zu stärken, um regieren und entscheiden zu können. Das gelingt ihm schliesslich.

In ihrem Einflechten von Feeding-Szenen in die Geschichte bleibt die Gruppe der Canon-Logik treu. Anders als Menschen können sich Wraith nicht für ihre Ernährungsweise entscheiden. Sie sind auf diese Nahrungsquelle angewiesen. Die Originalserie beschäftigt

8 Die Datumsangaben beziehen sich auf die im Stash-Gruppenarchiv gespeicherten Versionen der Episoden..

9 Bei der Namensgebung der Charaktere und dem Prinzip ihrer Gestaltung bezieht sich die Gruppe auf das Canon der Bücher zu Stargate Atlantis. 
sich häufiger damit, ob die DNA der Wraith nicht so verändert werden könne, dass sie sich anders ernähren. Das wäre also die Lösung aus Sicht der menschlichen Akteure. Im RP wird hingegen konsequent aus Wraith-Sicht gespielt und Empathie, wie in der besagten Episode, für die Belange und Bedürfnisse (hier: Hunger, Schwäche, Leistungsfähigkeit der Königin) der Wraith-Akteure erzeugt. Die primäre Funktion des Menschen ist, lebendig konserviert als Nahrungsmittel in der Vorrats- und Speisekammer zu lagern. Man sorgt sich um den Menschen so wie um eine knappe Ressource.

Es ist nicht so, dass in der Gruppe aus der Verhaltensanlage der Wraith weitschweifende ethische Debatten resultieren. Alles ereignet sich im belastbaren Wirklichkeitsmodus des Spielens. Gleichwohl sind Anschlussstellen für weiterführende Reflexionen gegeben: Der Mensch, der traditionell anderen Spezies die Plätze und Funktionen anweist, ist im RP selbst objektiviert und instrumentalisiert. In der selbstverständlichen Fortführung der Canon-Logik trotz der Perspektivumkehrung des RPs liegt die besondere Spannung: Als Menschen, die Wraith spielen, welche sich von Menschen ernähren, sind die Spielerinnen und Spieler gleichsam Herrschende und Beherrschte in Person-Figur-Union.

Solche in der Spielanlage des RP angelegten Potenziale der Befremdung zu elementaren Fragen der Existenzbedingungen (des Menschen wie anderer Spezies und Akteursgruppen) beschränken sich nicht auf das Thema Nahrung. Ein weiteres für Science-Fiction und das analysierte RP typisches Sujet besteht in der cyborgartigen Verwobenheit von biologischem Körper, Technik und Medien und der fragilen Abhängigkeit der Wraith von einer funktionierenden technologischen Infrastruktur.

Handlungsbezug: Niederknien, verbeugen oder rebellieren?

Die in den rund 200 im Beobachtungszeitraum entstandenen RP-Episoden in Gänze zu überblicken, ist kaum möglich. Jede einzelne Episode ist es Wert, auf ihre je spezifischen Beziehungs-, Ereignis- und 
Handlungsstrukturen und Dynamiken analysiert zu werden. Dies vorausgeschickt sei in Bezug auf Handlungsbezüge auf drei wiederkehrende und übergreifende Auffälligkeiten aufmerksam gemacht: zum einen auf die Grundspannung zwischen Blades und Clevermen untereinander, zum anderen zwischen beiden Gruppen und den Königinnen der Hives; und zum dritten auf die extensive Beschreibung der Innenwelten der Charaktere in Handlungs- und Bewährungssituationen.

Anders als im Canon begegnen sich Blades und Clevermen im für das RP zentralen Hive Fairweather in einer streng hierarchischen Beziehung, in der die Blades das Sagen haben. Diese Grundspannung durchzieht viele Episoden, unabhängig von deren Rahmenhandlung. Dramaturgisch ist diese Spannung ein Movens, das die Geschichte vorwärtstreibt, indem fortlaufend Konflikte entstehen.

Ein plotbestimmendes Ereignis, auf das schwach gerichtet ${ }^{10} \mathrm{zu}-$ gespielt wurde, ist die erwähnte Rebellion. Die Clevermen waren der schlechten Behandlung auf Fairweather überdrüssig und haben schliesslich das Angebot des verbündeten Hives Cyclone angenommen, dort unterzukommen und sich vor der Gewalt der Blades zu schützen. ${ }^{11}$ Vorausgegangen ist (unter anderem) eine Attacke auf

10 «Schwach gerichtet» weil das RP der Gruppe von Situativität und Spontaneität lebt. Besprochen und verabredet werden lediglich grössere Plotlinien sowie kurz vor den einzelnen Episoden deren Ausgangsdispositionen. Die prinzipielle Offenheit der Geschichte und die damit einhergehende Spannung (Wie geht es wohl weiter?) sind wichtige Motive, überhaupt zu spielen.

11 Die gegenläufige Namensgebung «Fairweather» (《Schönwetter») vs. «Cyclone» ("Wirbelsturm») ist ein bewusst gestaltetes Element des World Building der Gruppe. Laut Natalie spielt die Gruppe mit den so gesetzten Zuschreibungen aber auch. Eigentlich müsste das Leben auf Cyclone viel chaotischer und die Beziehungen «zerrütteter» sein. Die Geschichte beweist das Gegenteil. Fairweather spielt doppeldeutig zudem auf den Typus des «fair weather friend» an, also des «Schönwetterfreunds». Das kennzeichnet nicht unbedingt die tatsächlichen sozialen Beziehungen auf Fairweather, kann aber als Hinweis auf Konflikte unterhalb der Oberfläche gelesen werden. 
Luckless. In der Episode «Versed tries to get a report by Cinder + Luckless》 vom 20.06.17 sollen die beiden Clevermen Luckless (gespielt von Svenja) und Cinder (gespielt von Mario) dem Blade Versed (gespielt von Natalie) berichten, was genau bei dem Angriff geschehen ist. Die Episode illustriert gut, wie defensiv Clevermen gegenüber Blades agieren. Obgleich selbst Opfer des Angriffs, von dem zu diesem Zeitpunkt alle Charaktere noch annehmen, ein oder mehrere äussere Angreifer attackierten den Hive, entfalten die beiden ihren Bericht nur vorsichtig und zaghaft. Luckless hat eine Drohne gesehen, beeilt sich aber, etwaige kausale Ableitungen aus dieser Aussage zu relativieren: «But I don't want to go as far and say this leads to anything. I just didn't want to keep that information away». Diese zurückhaltende Art ist nicht nur ein persönlicher Charakterzug von Luckless. Schon in den ersten Episoden, in denen Svenja aktiv mit Luckless spielt, wird ihm als Charakter (und Svenja als Spielerin) deutlich gemacht und spüren gelassen, dass man sich in der Interaktion mit Blades besser nicht zu weit aus dem Fenster lehnt. Umgekehrt ist der Ton der Blades den Clevermen gegenüber oft von direktiven Ansprachen und Befehlen gekennzeichnet. Dass das RP bei dieser Asymmetrie nicht stehenbleibt, zeigt die Rebellion und die nachfolgende Flucht der Clevermen, die zwischen der oben und hier zitierten Episode stattfinden.

Hierarchische Beziehungen und die Fragen, welche Handlungsund Gestaltungsräume bestehen und wie weit Grenzen verschoben und in Frage gestellt werden können, ziehen sich durch das gesamte RP, nicht nur im Verhältnis von Blades und Clevermen. Ein Handlungsstrang der oben genannten Episode um Wallflower (Queen) und Deepwater (Cleverman) besteht im Vertrautwerden der beiden, wohl wissend, dass damit Konventionen verletzt werden. Deepwater steht nicht nur hierarchisch unter der Queen, sondern gehört einem anderen Hive an. In der sich anbahnenden Beziehung (als Lesende werden wir im Ungefähren gelassen über deren potenziell romantischen Charakter) sind Grenzverletzungen auf verschiedenen Ebenen impliziert. Eine Szene innerhalb der Episode besteht in 
der Modulation bzw. dem Wechsel des üblichen Verhaltensrahmens. Wallflower fordert Deepwater auf, nicht zu knien. Übersetzt bedeutet das, die üblicherweise notwendigen Ehrerbietungsrituale zu unterlassen. Gleichwohl bleibt der Spielraum beschränkt: «A simple bow suffices». Es schliesst sich eine (auch aus Figurenperspektive) spassig kodierte Szene an, in der Deepwater zur Belustigung der Queen verschiedene Weisen des Verbeugens durchspielt - «testing the bounds somewhat».

Wallflower ist in punkto Hierarchie auch darüber hinaus ein interessanter Charakter. Nach dem Tod der alten Königin ist sie noch nicht lange Queen von Fairweather. In vielen Episoden wird ihr Innen- und Seelenleben beschrieben. Immer wieder tritt ihre Unsicherheit zu Tage. Oft weiss Wallflower nicht, ob sie in der Rolle der Queen angemessen agiert, fragt sich, ob sie zu weich auftritt und ihre Untertanen den Respekt verlieren, wenn sie sich nicht den antizipierten Gepflogenheiten entsprechend verhält. Gleichzeitig scheint sie sich das starre Korsett des antizipierten Anforderungsprofils einer führungsstarken und erfolgreichen Königin nicht anziehen zu wollen und einen dritten, eigenen Weg zu suchen.

Die Beschreibung der Gedankenwelt, das Hadern mit den sozialen Verhältnissen und Rangordnungen, das Spiegeln in den antizipierten und interpretierten Deutungen und Reaktionen der Anderen nimmt im RP breiten Raum ein. Nur selten lösen sich die Ambivalenzen in einem Happy End auf. Vielmehr sind sie spielbestimmend und sind Teil des World Buildings der Gruppe. Das Leben der Wraith in der Pegasus-Galaxie, es ist kompliziert.

\section{Wissen und Biografie: Komplexe statt glatte Charaktere!}

Wissen erschliessen, aneignen und reproduzieren ist eine Grundbedingung des RP-Spielens. Ohne umfangreiches Wissen sind die Autorinnen und Autoren nicht spielfähig. Für Svenja bestand die Schwierigkeit zu Beginn nicht nur darin, sich Basiswissen zum 
transmedial erzählten Canon anzueignen, also die Serie zu schauen, Bücher zu lesen und Fan-Wikis zu studieren. Zusätzlich musste sie sich auch in das komplexe Netz an Figuren, Beziehungen und Handlungssträngen als gruppenspezifisches Fanon einarbeiten, das die Gruppe vor ihrem Eintritt bereits etabliert hatte. Sich kompetent in der Geschichte bewegen zu können, ist wichtig. Wer zu wenig vom Canon und Fanon weiss, kommt im Wortsinn nicht mit und stört den Fluss des Spiels.

Bildungsbezogen sind vor allem jene Momente relevant, in denen subjektive Wissensaufschichtungen in den Fluss geraten, sich relativieren oder konkurrieren. Inwiefern das in den Köpfen und Körpern der Spielerinnen und Spieler geschieht oder sich eigene biografische Erfahrungen im Schreibspiel manifestieren, kann auf der Basis der vorliegenden Daten nur ansatzweise nachvollzogen werden. Gleichwohl ist auch solchen Prozessen in mindestens zweifacher Weise der Nährboden bereitet:

Zum einen ist in spielmethodischer Hinsicht die Diskrepanz von Spielerwissen und Figurenwissen konstitutiv. Die Figuren wissen prinzipiell weniger als die Spielerinnen und Spieler. Mithin besteht der Reiz gerade darin, die Charaktere mit den nächsten Ereignissen, Krisen, Dramen und Volten zu konfrontieren und zu schauen, wie diese für sich und in Interaktion miteinander umgehen und was sich daraus wiederum für den Fortgang der Geschichte ergibt. Die Diskrepanz von Autor-/Spieler-Wissen und Figurenwissen betrifft freilich ebenso andere Formen des literarischen Schreibens. Dadurch, dass die Story im RP aber prinzipiell ergebnisoffen und situativ vorangetrieben wird, überraschen sich die Spielerinnen, Spieler und Figuren immer wieder auch gegenseitig mit unvorhergesehenen Handlungs- und Verhaltensmustern und Wendungen. Zumindest das RP dieser Gruppe führt gewissermassen zwangsläufig dazu, Wissensgefüge als relativ, vorläufig und in Bewegung zu betrachten. 
Zum anderen sind Irritationen und Transformationen von Orientierungen und Wissensbeständen auf der Ebene der Figuren selbst angelegt. Ihre Erfahrungen und ihre Entwicklung stehen oft im Mittelpunkt. Die Begegnung zweier einander fremder oder noch wenig bekannter Charaktere führt nicht selten zu längeren biografischen Dialogen. In der oben vorgestellten Episode um Wallflower und Deepwater regt die Queen den Cleverman an, ihr seine Geschichte zu erzählen. In diesem Fall mündet das in einen hypothetischen Dialog, warum Deepwater kein Blade geworden sei, worauf wiederum das Thema Hierarchie und Macht Eingang in das Gespräch findet. Er habe sich immer auf seine Arbeit als Techniker und Wissenschaftler konzentriert und sich aus den «decision-making ranks» herausgehalten. Er habe gesehen, was Macht aus Wraith machen kann und wie einsam Macht macht: «It seems lonely». In der sich anschliessenden Passage lenkt Deepwater das Gespräch in eine andere Richtung: «\{By the way, $\}$ he said, \{what is your hive's policy on worshippers?\}».12 Wallflower gibt sich unwissend, was Worshippers sind (siehe Abschnitt Grenzbezug) und lässt sich aufklären. Als Leserinnen und Leser wohnen wir einem Irritationsmoment bei. Wallflower registriert mit Interesse, dass Deepwater die menschliche Frau an Bord seines Schiffes selbst entscheiden lassen würde, ob diese mit ihm zu Wallflowers' Hive käme. Hierarchisch betrachtet hätte sie der Anweisung des Wraith einfach Genüge zu leisten. Diese kleine, nicht weiter ausgeführte Passage reiht sich ein in das Mosaik an Erfahrungen und Selbstreflexionen, die Wallflower zu der Person machen, die sie ist: sensibel und offen für Orientierungen, Dinge ein wenig anders zu machen und zu handhaben.

Die NPCs (non-playable characters) ausgenommen wissen beinahe alle Hauptfiguren von krisenhaften Ereignissen und wechselhaften Biografien zu berichten. Komplexe Charaktere, die sich einer einfachen Gut-/Böse-Dichotomie entziehen, dominieren das RP.

12 Wraith sind telepathische Wesen. Die Gruppe nutzt geschweifte Klammern, um telepathische Kommunikation zu kennzeichnen und doppelte Anführungszeichen für verbale Kommunikation. 


\section{Abschliessende Diskussion und Reflexion}

Durch die Brille des Medienbildungsansatzes wurde in diesem Beitrag auf ein literarisches RP geschaut, das in einem anderen Forschungszusammenhang entstanden ist, sich aber für eine bildungsbezogene Relektüre anbietet. Abschliessend sollen die exemplarisch herausgearbeiteten Medienbildungspotenziale eine reflektierende Einordnung erfahren, auch selbst-reflexiv mit Blick auf eigene Orientierungen, die sich in die Deutung einschreiben.

Fan Studies wird oft (und manchmal zu Recht) der Vorwurf gemacht, ihren Gegenstand zu zelebrieren und die kulturelle und gesellschaftliche Bedeutung von Fanhandeln zu überhöhen. Dass die Partizipation und eigentätiges produktives Handeln von Fans wertvoll sind, wird nicht selten damit begründet, dass sich Fans bilden bzw. Wissen, Kompetenzen, Fähigkeiten und Fertigkeiten erwerben, die hilfreich sind für das (Über-)Leben in der Mediengesellschaft. $\mathrm{Ob}$ das der Fall ist, hängt aber nicht allein davon ab, was Fans tatsächlich tun und erfahren, sondern ebenso davon, wie Forschende Bildung (und Kompetenz) definieren und welche expliziten und impliziten Prämissen sie einführen. Im Rahmen des Diskurses um Medienbildung betrifft das die «Fallhöhe», ab wann eigentlich von einer «grundlegenden» Transformation des Selbst- und Weltverhältnisses zu sprechen sei, ebenso wie die Frage, ob Bildung per se in etwas «Neues» münden müsse (oder mitunter nicht Beharrung gelegentlich die bessere Bildung sei), und ob die Richtung von Bildungsprozessen seitens der Forschung nicht normativ zu bewerten und mehr oder weniger wünschenswerte Transformationen zu unterscheiden seien (Koller 2016).

Die Ausführungen zum hier ausschnitthaft porträtierten und analysierten Wraith-RP verdeutlichen zunächst, dass im RP-Spielen/Schreiben permanent Perspektivierungsarbeit vollzogen wird. In der Logik der hier vorgetragenen Argumentation ist damit zumindest eine wichtige Voraussetzung für Bildungsprozesse erfüllt. Perspektivierungsarbeit ist freilich ebenso für andere Formen der 
literarischen Textproduktion konstitutiv. Das Verfassen fiktionaler Texte impliziert beinahe immer, aus der Warte von Charakteren zu denken, zu fühlen, sie in Ereignisse, Handlungsketten und in Welten zu platzieren, die selbst wiederum gestaltet werden. Die dialogische Grundsignatur von RP und das kollektive Verfassen von Texten geht über diese Disposition jedoch hinaus. Das für interpersonale Kommunikation charakteristische, wechselseitige Zuführen und Einnehmen von Perspektiven in einem per se offenen, nicht absehbaren Prozess verbindet sich hier mit medientextuellen Perspektivierungsarbeiten, wie sie in der Medienkulturproduktion von Spiel-, Schrift- und anderen Werken stets zu leisten sind.

Mit Blick auf die normative Valenz meiner/unserer Deutungen ist eine tendenziell optimistische, in Teilen anwaltliche Interpretationsrichtung der Geschichtengehalte nicht zu leugnen. Das liegt einerseits an der Kenntnis der spielenden Personen, begründet sich andererseits aus dem Wissen darum, dass Fan Fiction-Autorinnen, -Autoren sowie RP-Spielerinnen und -Spieler noch heute in ihrem Interesse oft belächelt werden. Wir hingegen sind beeindruckt von der immensen kreativen Energie, die die Gruppe im Spielmodus freisetzt. Freilich aber steht es jeder und jedem frei, zu anderen Schlüssen zu kommen. Wo ich/wir das RP als eine kritische Beschäftigung mit Hierarchie und Macht deuten, könnte gegenläufig z.B. gefragt werden, warum, wo doch die Möglichkeit besteht, nicht eine alternative, utopische Welt ohne diese Machtgefälle geschaffen wurde. ${ }^{13}$

Unabhängig von der Valenz der Deutung schliesst sich die bildungsbezogene Frage an, ob sich durch das Spielen dieses RPs oder durch RP-Spielen generell die Selbst- und Weltverhältnisse der Autorinnen und Autoren wandeln. Die Begleitinterviews zur Gruppe unterstreichen die grosse Bedeutung von RP im Alltag und der Lebensgeschichte der Spielerinnen und Spieler, die sich ebenso abseits des Spiels ständig mit möglichen Szenarien und Fortführungen der

$13 \mathrm{Um}$ eine banale, aber treffende (Teil-)Antwort gleich mitzuliefern:

Perfekte Welten eignen sich nicht zum Spielen. Es braucht Spannung,

Konflikt und Kontroverse. 
Geschichte beschäftigen. Es liegt nahe, dass das permanente Jonglieren, Bearbeiten und Ausgestalten von und mit Figuren, Ereignissen, Handlungsketten und Welten Spuren hinterlässt. Vermutlich blicken die Autorinnen und Autoren tatsächlich anders auf sich und die Welt als sie es ohne diese - in der hier portraitierten Gruppe seit vielen Jahren gelebte - Medienpraxis tun würden. Was diese Praxis mit jeder/jedem Einzelnen genau macht und gemacht hat, in welcher Weise sie sich mit und an ihr gebildet haben, können wir (in diesem Projekt, mit anderem Fokus) nur bedingt nachvollziehen. Arbeiten in den Fan Studies, die mit Performance-Ansätzen argumentieren allen voran Lancaster (2001) mit seiner Pionierarbeit zu Babylon 5 stehen vorschnellen Transfer-Thesen allerdings ohnehin skeptisch gegenüber (nicht zuletzt in Folge der langatmigen Stigmatisierung von Fanhandeln in den diversen Gewalt-, Nerd- und Eskapismusdiskursen). Demnach ist das Spielen, Imaginieren und Fantasieren als Wirklichkeitsmodus eigenen Rechts ernst zu nehmen, auch dahingehend, nicht immer gleich umfassende Lern- und Bildungsprozesse am Werke sehen zu wollen, die auf andere Modalitäten des In-derWelt-Seins und andere Lebensbereiche ausserhalb des Spiels ausstrahlen (was selbstverständlich nicht ausgeschlossen ist, kommt es doch auf die Präge- und Irritationskraft von - wo immer - gemachten Erfahrungen an). Diesen Gedankengang weiterführend liegt neben dem Potenzial von RP und anderen Faneigenproduktionen, selbst-reflexive Prozesse zu stimulieren, ein bildungsbezogen relevantes Momentum im übergreifenden Enskillment, Perspektivierungsarbeiten vorzunehmen. Entsprechende Fähigkeiten sind nicht schon selbst die Bildung (und gehen über Medienkompetenz im engeren Sinn hinaus), aber bahnen ihr gewissermassen den Weg, machen sie wahrscheinlicher. Dafür jedenfalls ist RP-Spielen ein Trainingsparcours par excellence. 


\section{Literatur}

Black, Rebecca W. 2008. Adolescents and online fan fiction. New York et al.: Peter Lang.

Cuntz-Leng, Vera, und Jacqueline Meintzinger. 2015. "A brief history of fan fiction in Germany》. Transformative Works and Cultures 19. https:// doi.org/10.3983/twc.2015.0630.

Hine, Christine. 2015. Ethnography for the Internet: Embedded, Embodied and Everyday. Huntingdon: Bloomsbury.

Jenkins, Henry, Ravi Purushotma, Margaret Weigel, Katie Clinton, und Alice J. Robison. 2006. Confronting the Challenges of Participatory Culture: Media Education for the 21st Century. Chicago: The John D. and Catherine T. MacArthur Foundation. Zugriff 16.09.2012. http://www. digitallearning.macfound.org.

Jörissen, Benjamin, und Winfried Marotzki. 2009. Medienbildung - eine Einführung: Theorie - Methoden - Analysen. Bad Heilbrunn: Julius Klinkhardt.

Knobel, Michele, und Colin Lankshear. 2008. «The Art and Craft of Endless Hybridization». Journal of Adolescent \& Adult Literacy 52 (1): 2233.

Koller, Hans-Christoph. 2016. «Ist jede Transformation als Bildungsprozess zu begreifen? Zur Frage der Normativität des Konzepts transformatorischer Bildungsprozesse». In Von der Bildung zur Medienbildung. Festschrift für Winfried Marotzki, herausgegeben von Dan Verständig, Jens Holze, und Ralf Biermann, 149-61. Wiesbaden: Springer VS.

Lancaster, Kurt. 2001. Interacting with Babylon 5. Fan Perfomances in a Media Universe. Austin: University of Texas Press.

\section{Acknowledgement}

Die in diesem Artikel präsentierte Forschung ist im Rahmen des Projekts Bo7 «Medienpraxis und Urheberrecht» des von der DFG geförderten Sonderforschungsbereichs 1187 «Medien der Kooperation» an der Universität Siegen entstanden. 